\title{
Results from ATLAS and CMS: Strong Interactions and New Physics
}

\author{
Siegfried Bethke ${ }^{* \dagger}$ \\ Max-Planck-Institute of Physics, Munich \\ E-mail: bethke@mpp.mpg •de
}

\begin{abstract}
Measurements on global properties and precision results on fundamental parameters related to the Strong Interaction sector of the Standard Model of particle physics, and searches for new phenomena beyond the Standard Model, performed by the two large multi-purpose particle detectors at the Large Hadron Collider (LHC), are summarised in this review. Special attention is payed to the new data obtained at $\sqrt{s}=13 \mathrm{TeV}$ in 2015, which offer a first glimpse at the large physics potential offered by the high-energy running of the LHC.
\end{abstract}

54th International Winter Meeting on Nuclear Physics

25-29 January 2016

Bormio, Italy

* Speaker.

${ }^{\dagger}$ On behalf of the ATLAS and CMS collaborations. 


\section{Introduction and LHC Operation}

The Large Hadron Collider (LHC) [1] is the world's largest and most powerful particle accelerator, located in a ring-shaped underground tunnel of $27 \mathrm{~km}$ circumference. Built and operated by the European Laboratory for particle physics, CERN, in the vicinity of Geneva, Switzerland, the LHC accelerates, stores and collides two beams of protons at c.m. energies $(\sqrt{s})$ of up to $13 \mathrm{TeV}$ or - alternatively - beams of lead ions up to nucleon-nucleon c.m. energies, $\sqrt{s_{N N}}$, of $5.00 \mathrm{TeV}$ (equivalent to $\sqrt{s_{P b P b}}$ of $1.1 \mathrm{PeV}$ ). For short amounts of time and within regions of space-time limited by the quantum size of the colliding objects, these collisions create energy densities that closely resemble the conditions of the very early, hot and dense universe, at only $10^{-10}$ to $10^{-15}$ seconds after the Big Bang.

From 2009 to 2012, LHC provided integrated luminosities of about $5 \mathrm{fb}^{-1}$ at $\sqrt{s}$ of $7 \mathrm{TeV}$, and of $25 \mathrm{fb}^{-1}$ at $\sqrt{s}$ of $8 \mathrm{TeV}$. This period is commonly referred to as run-1. After the first long shutdown in 2013/2014 and more than $10^{6}$ working hours spent for maintenance and the energy upgrade of LHC, proton collisions at $\sqrt{s}$ of $13 \mathrm{TeV}$ were established in 2015, with an integrated luminosity of about $5 \mathrm{fb}^{-1}$ for each of the two large multi-purpose detectors, ATLAS (Fig. 1) and CMS . This running period called run-2 is scheduled to commence until the end of 2018, reaching integrated luminosities of about $100 \mathrm{fb}^{-1}$ at $\sqrt{s}$ of 13 and later $14 \mathrm{TeV}$. These data will provide a wealth of precision results on the Standard Model of Particle Physics (SM) and beyond (BSM), at the highest accessible collider energies.

In this presentation, I will focus on recent results from ATLAS and CMS on topics of the strong interaction, the part of the SM dominating most physics processes at LHC, and on searches for BSM physics. Wherever possible, first results from the most recent run-2 data at $\sqrt{s}=13 \mathrm{TeV}$ will be shown with preference. Topics related to electroweak interactions and symmetry breaking, and beyond, will be covered by P. Azzurri at this conference [3].

\section{The ATLAS Detector}

The ATLAS detector [4] is one of the two large multipurpose particle detectors ${ }^{1}$ operated at the LHC. It was designed, built, and is being operated by an international collaboration of more than 3000 scientists from 178 institutes and 38 countries world-wide. With its length of $44 \mathrm{~m}$, a diameter of $25 \mathrm{~m}$ and a weight of 7000 tons, ATLAS is one of the largest but rather light (in terms of weight/volume) particle detectors in the world. Symmetrically built around one of the proton beam collision points of LHC, ATLAS records the debris of the collision products of proton-proton and lead-lead collisions with highest precision, in order to reconstruct the dynamics and nature of the elementary collision reactions.

Within ATLAS, secondary particles, created by the collisions which leave the collision area and the LHC beampipe, are recorded by a number of different particle detector types. The ATLAS charged particle tracker, consisting of layers of silicon pixel and strip detectors plus outer shells of transition radiation detectors, allows to precisely measure the tracks of charged particles in a surrounding 2-Tesla solenoidal magnetic field, to determine the particle's momenta and to reconstruct

\footnotetext{
${ }^{1}$ The second multipurpose-detector, CMS [5] is described in more details in P. Azzurri's presentation [3] at this conference.
} 
the location of their origin, namely one of the primary interactions of protons along the nominal beam direction, or possible secondary vertices from decays of heavy, short-lived objects like bquarks. Electromagnetic and hadronic calorimeters around the tracker provide measurements of the energies of particles and particle jets, followed by a large muon spectrometer that detects and precisely measures the trajectories of muons in the outer magnetic field, generated by a system of large superconducting toroidal magnet coils.

The hermeticism of the ATLAS detector, both perpendicular as well as parallel to the LHC beam direction, allows to also reconstruct and detect particles which normally leave no trace in particle detectors, like neutrinos or new particles beyond the Standard Model (BSM). The granularity and optimised resolution of the detector, consisting of more than 150 million electronic channels and a dedicated multi-step system of triggers and data reduction, allows to record all relevant particle reactions at $40 \mathrm{MHz}$ collision rates without sizable losses caused by dead-times and processing times. The ATLAS readout and trigger system allows to filter and reduce the initial raw data flux of up to $10^{16}$ bytes per second ${ }^{2}$ (bps) to a finally recorded data rate of a few hundred collisions per second, or equivalently, up to one Gigabyte of data per second. These data are sent to the CERN Tier- 0 computing centre, stored, calibrated and distributed to the world-wide LHC computing grid (WLCG), consisting of several 100 computing centres around the globe, for further processing, storage and physics analysis.

One of the highest mass di-jet events, measured and reconstruted by ATLAS at $\sqrt{s}=13 \mathrm{TeV}$, is shown in Fig.2.
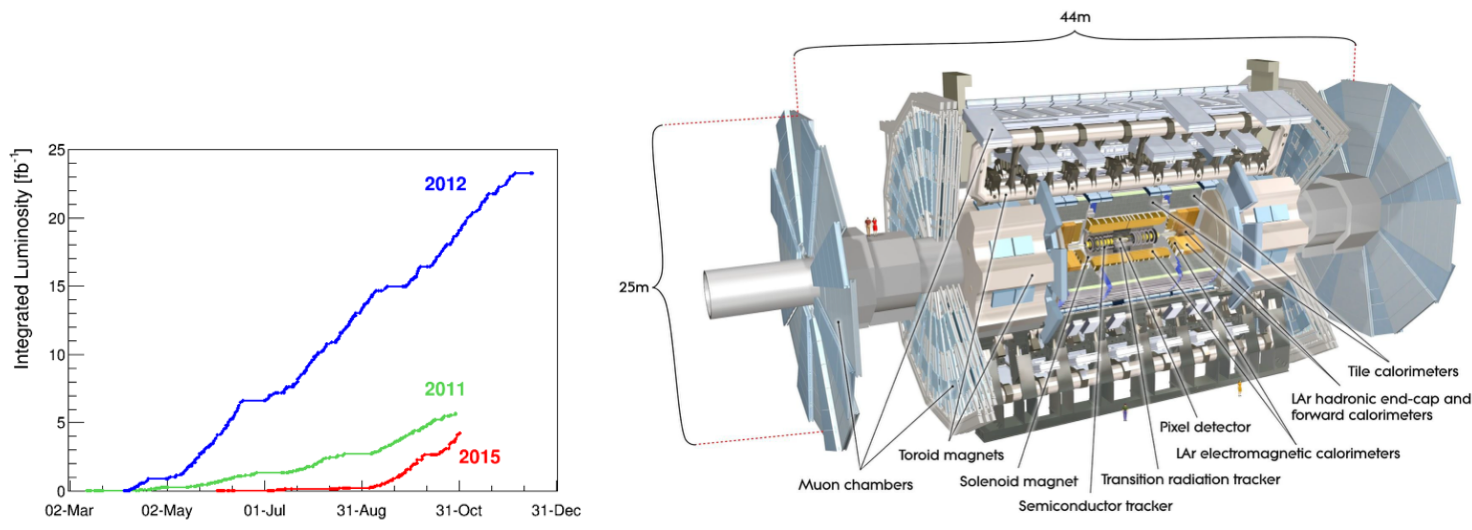

Figure 1: History of the integrated luminosity [2] (left) and structure of the ATLAS detector [4] operated at the Large Hadron Collider (right).

\footnotetext{
${ }^{2}$ Design values of LHC operation are: $40 \mathrm{MHz}$ collision rate of $\mathrm{p}$-p bunches with $10^{11}$ protons per bunch, corresponding to one collision every $25 \mathrm{~ns}$, at a luminosity of $10^{34} \mathrm{~cm}^{-2} \mathrm{~s}^{-1}$. A luminosity of $70 \%$ of the design value was routinely achieved in the 2015 run. $40 \mathrm{MHz}$ bunch crossing rate with a pile-up of typically 13 single p-p collisions per bunch crossing generates a raw data flux of up to $10^{16}$ Bytes per second, which is equivalent to the transmission of 10 billion telephone calls at a given time.
} 


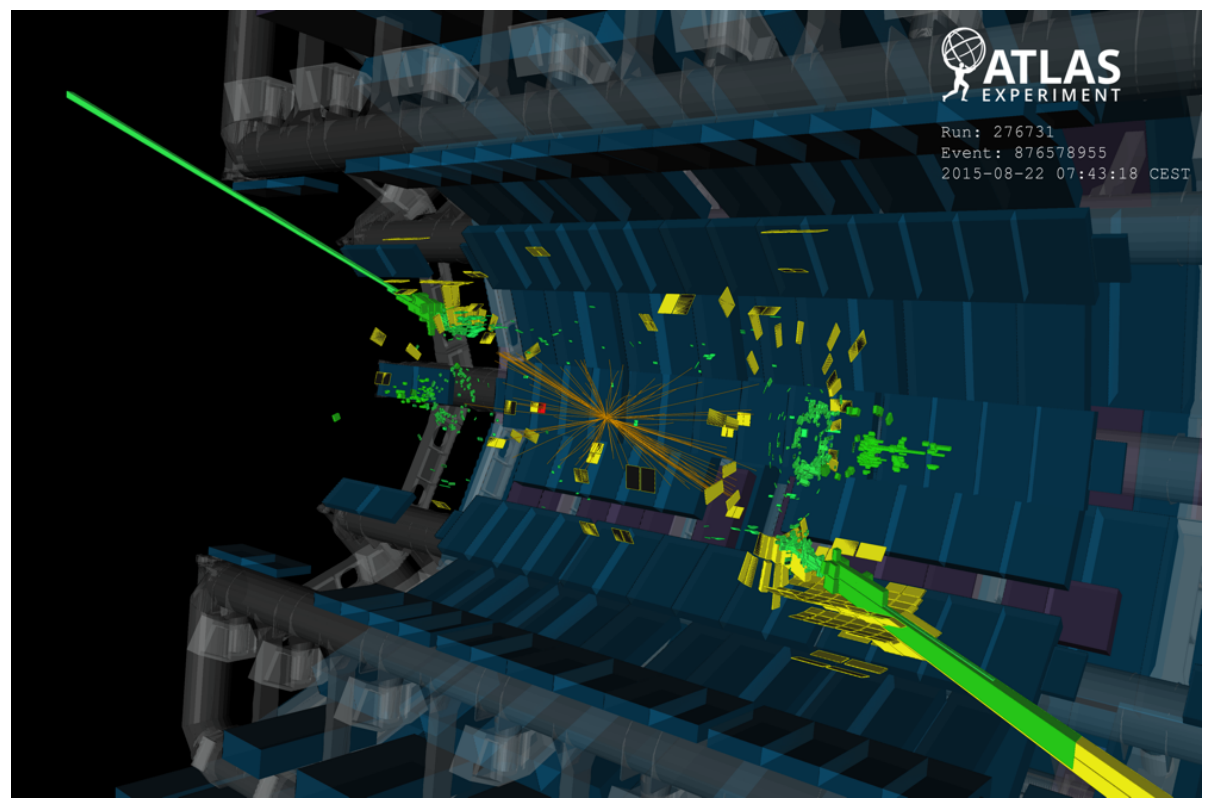

Figure 2: A high-mass di-jet event recorded at $\sqrt{s}=13 \mathrm{TeV}$. The two high- $p_{T}$ jets have an invariant mass of $6.9 \mathrm{TeV}$ and the leading and subleading jet have a $p_{T}$ of 1.3 and $1.2 \mathrm{TeV}$ respectively.

\section{Production Cross Sections at the LHC}

At LHC energies, i.e. at collision energies around $10 \mathrm{TeV}$, the total cross section for protonproton collisions is about $10^{8} \mathrm{nb}=10^{-25} \mathrm{~cm}^{2}$, which is totally dominated by the strong interaction between quarks and gluons, the basic constituents of hadronic matter. This corresponds, at LHC design luminosity, to $10^{9}$ elementary collisions per second, which is about six orders of magnitude higher than the expected production rate of $\mathrm{W}$ and $\mathrm{Z}$ bosons, and nine orders of magnitude higher than Higgs boson production [6] or other objects with masses beyond $100 \mathrm{GeV} / \mathrm{c}^{2}$, see Fig. 3 (left).

While most of the "standard" strong interaction background is filtered away by the trigger system described above, dedicated searches for and studies of $\mathrm{W}$ and $\mathrm{Z}$ bosons, of Higgs bosons and signals for new BSM physics, are based on further selection criteria like the presence of energetic leptons (electrons or muons), photons, hadron jets, secondary decay vertices or missing transverse (w.r.t. the beampipe) energy. These, or subsets of these signatures, indicate the decay of vector bosons ( $\mathrm{Z}$ and $\mathrm{W}$ ), or other heavy objects like Higgs bosons, top-quarks or new BSM particles. Such objects are then used to perform precision studies and determinations of the Standard Model and its fundmental parameters like masses and couplings, and to search for signatures for BSM physics.

The production cross sections for massive objects usually increase with increasing c.m. energy of the collider, see Fig. 3. The actual gain in cross sections at the highest LHC energy of $13 \mathrm{TeV}$, compared to the previous running at $8 \mathrm{TeV}$, is given in the right part of Fig.3. While the total hadronic cross section ("Min. bias" in Fig.3) only midly increases with $\sqrt{s}$, the production cross sections of SM particles like vector bosons, the Higgs boson and the top quark, increase by factors of 2 to 4 . Cross sections for new BSM particles like supersymmetric quarks and gluons, heavier 
vector bosons and other objects, however, gain even more from the increased LHC energy of LHC Run-2, by factors around and exceeding 10 . Note that already now, with the integrated luminosity available from the first year year of Run-2 data taking, some of the gains of cross sections already exceed the luminosity-weighted cross section which is marked, for CMS, by the vertical line.
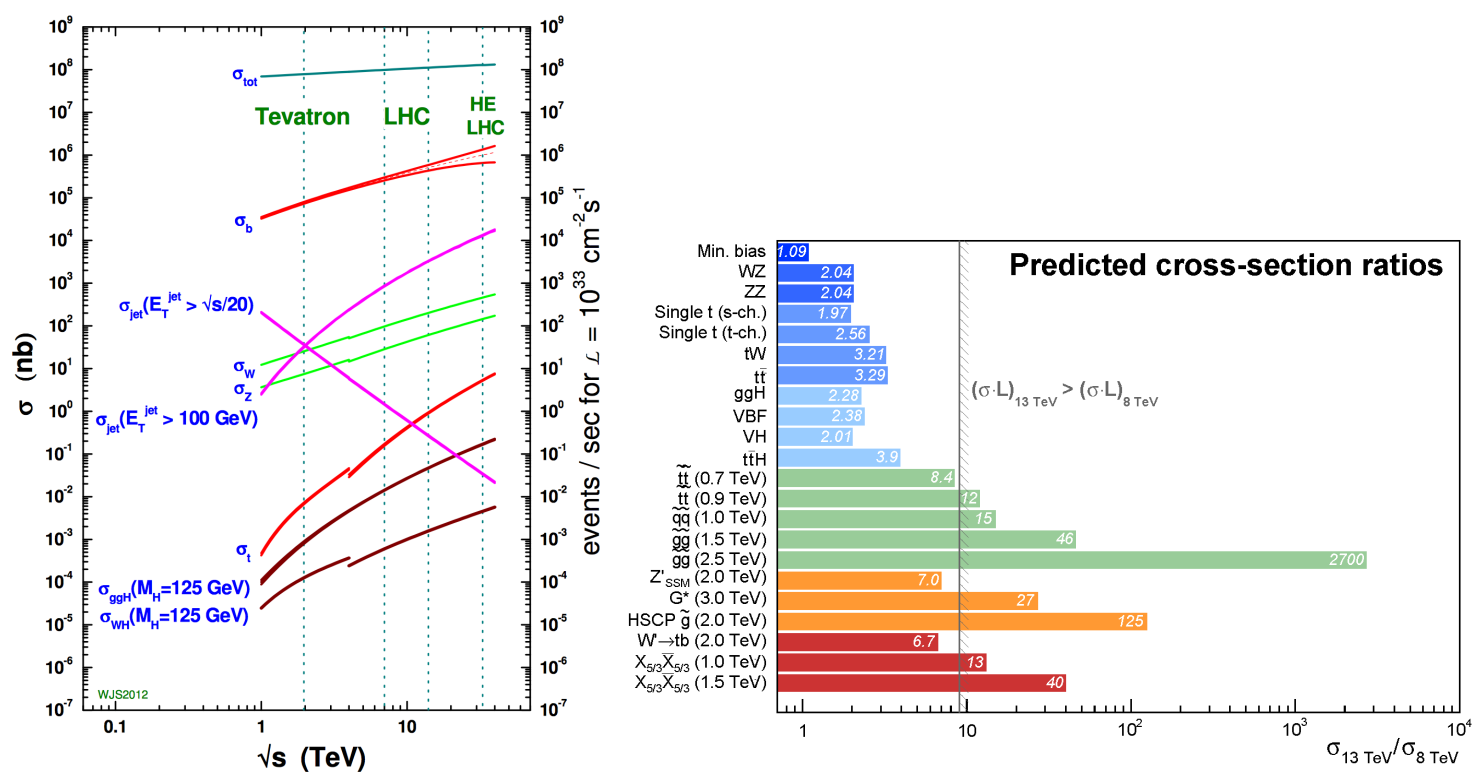

Figure 3: Left: Production cross sections at Tevatron and at LHC [6]. Right: ratios of production cross sections at LHC c.m. energies of 13 and $8 \mathrm{TeV}$; the vertical line indicates the luminosity-weigthed cross sections for CMS at the end of 2015 data taking [7]

\subsection{Inelastic p-p cross section}

A measurement of the inelastic total proton-proton cross section was performed by ATLAS [8] at $\sqrt{s}=13 \mathrm{TeV}$, using Minimum Bias Trigger Scintillators (MBTS) mounted in front of their forward calorimeter, resulting in $\sigma_{\text {inel. }}=73.1 \pm 0.9$ (exp) \pm 6.6 (lumi) \pm 3.8 (extrapol.) $\mathrm{mb}$. A comparison with previous measurements, at lower c.m. energies as well as one from cosmic rays at higher energies, is presented in the left part of Fig. 4. The data agree with the predictions by model calculations; a future measurement with higher precision may well differentiate between models.

\subsection{Charged Particle Multiplicities and Correlations}

Further studies of global properties of hadronic final states at LHC comprise the measurements of charged particle multiplicities and their correlations. A measurement of the mean charged particle multiplicity of inelastic p-p scattering events is shown in Fig. 5 (left), demonstrating that the energy dependence of this basic quantity is well described by model calculations, inspired by Quantum Chromodynamics (QCD) [9], but also by a simple parabolic fit in $\ln (\mathrm{s})$ [11]. The right part of Fig.5 shows a measurement [12] of two-particle correlations in $\eta-\phi$ space within high-multiplicity p-p events, where $\phi$ is the azimuthal angle around the beam pipe, and $\eta$ is the pseudo-rapidity, a measure of the angle w.r.t. the beam axis. These results confirm the presence of 


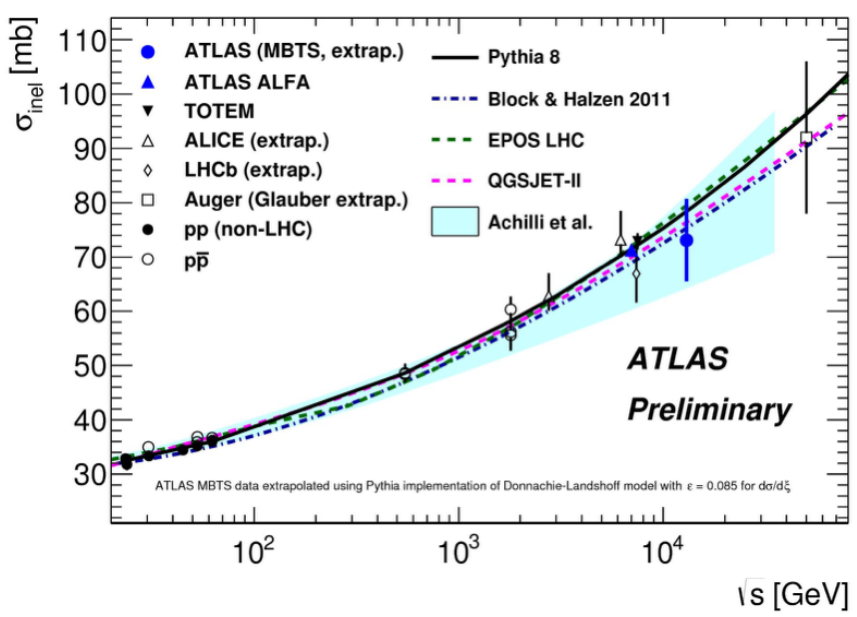

Figure 4: Inelastic p-p cross sections as function of the collider c.m. energy [8].

a ridge-like structure for same-side $(\Delta \phi=0)$ pairs in high-multiplicity events at $13 \mathrm{TeV}$ c.m. energy, which is also seen by ATLAS [13]. One might speculate that this is an effect of color-strings connected between the hard scattered quark (or gluon) and the proton remnants of the collisions.
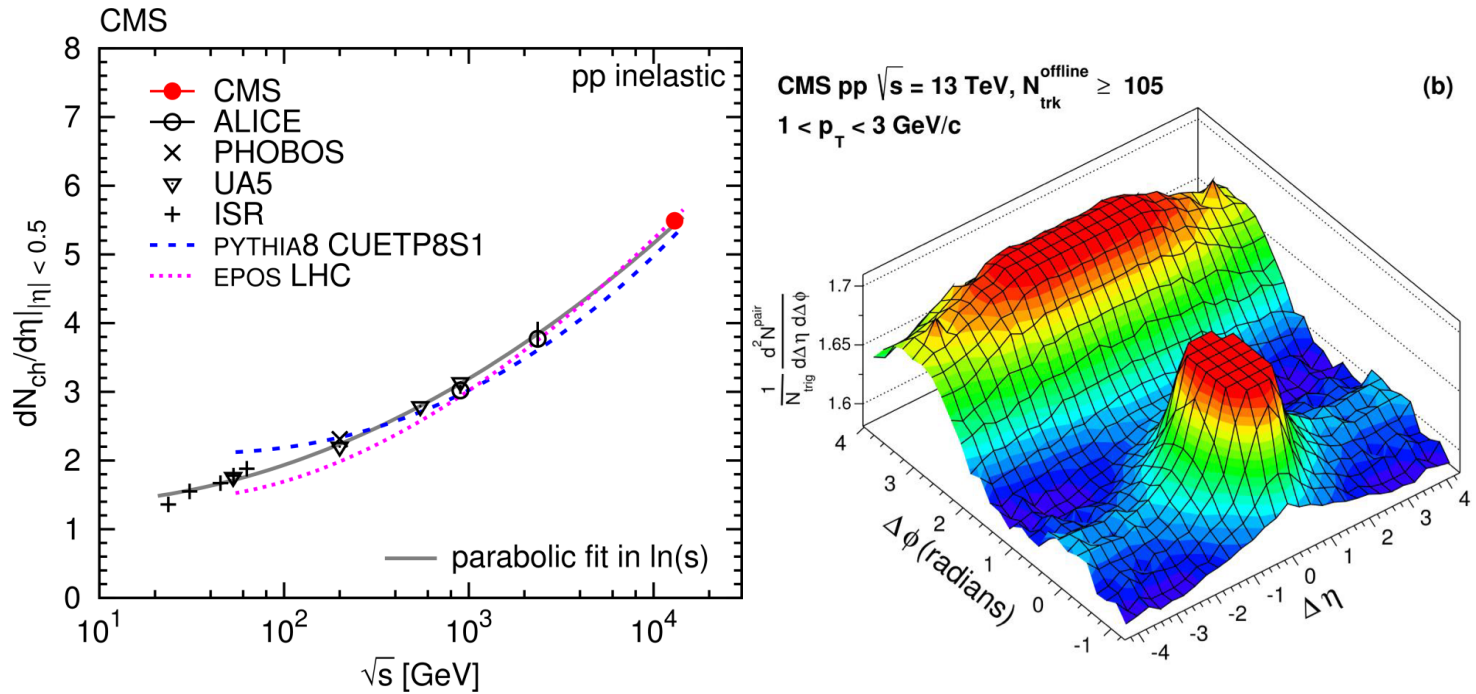

Figure 5: Mean charged particle multiplicities of inelastic proton-proton collisions as function of the collision c.m. energy [11] (left); two-particle correlations of charge particles within high multiplicity protonproton collisions at $\sqrt{s}=13 \mathrm{TeV}$ [12] (right) demonstrating presence of the "ridge-effect", previously discovered in lead-lead-collisions, for same-side particle pairs $(\Delta \phi \approx 0)$.

\subsection{Inclusive Jet Cross Sections}

The next level of specific properties of hadronic final states are measurements of inclusive jet cross sections, which constrain the underlying parton desity functions (pdf) and are predicted 
by perturbative QCD calculations. Fig.6 shows such cross sections, as a function of transvers jet momentum $\left(\mathrm{p}_{T}\right)$ and in different bins of pseudo-rapidity, measured by CMS using initial data from the 2015 run at $\sqrt{s}=13 \mathrm{GeV}$ [14]. Jets are defined using a specific jet definition (in this case, the anti- $\mathrm{k}_{t}$ algorithm [15]) with a resolution parameter of $\mathrm{R}=0.4$. Similar as observed at lower c.m. energies, QCD predictions, based on a specific set of parton pdf's, describe the data well, over many orders of magnitude of cross sections and in the $\mathrm{p}_{T}$-range of up to $2 \mathrm{TeV}$. The agreement using alternative sets of pdf's is depicted in the right part of Fig.6.
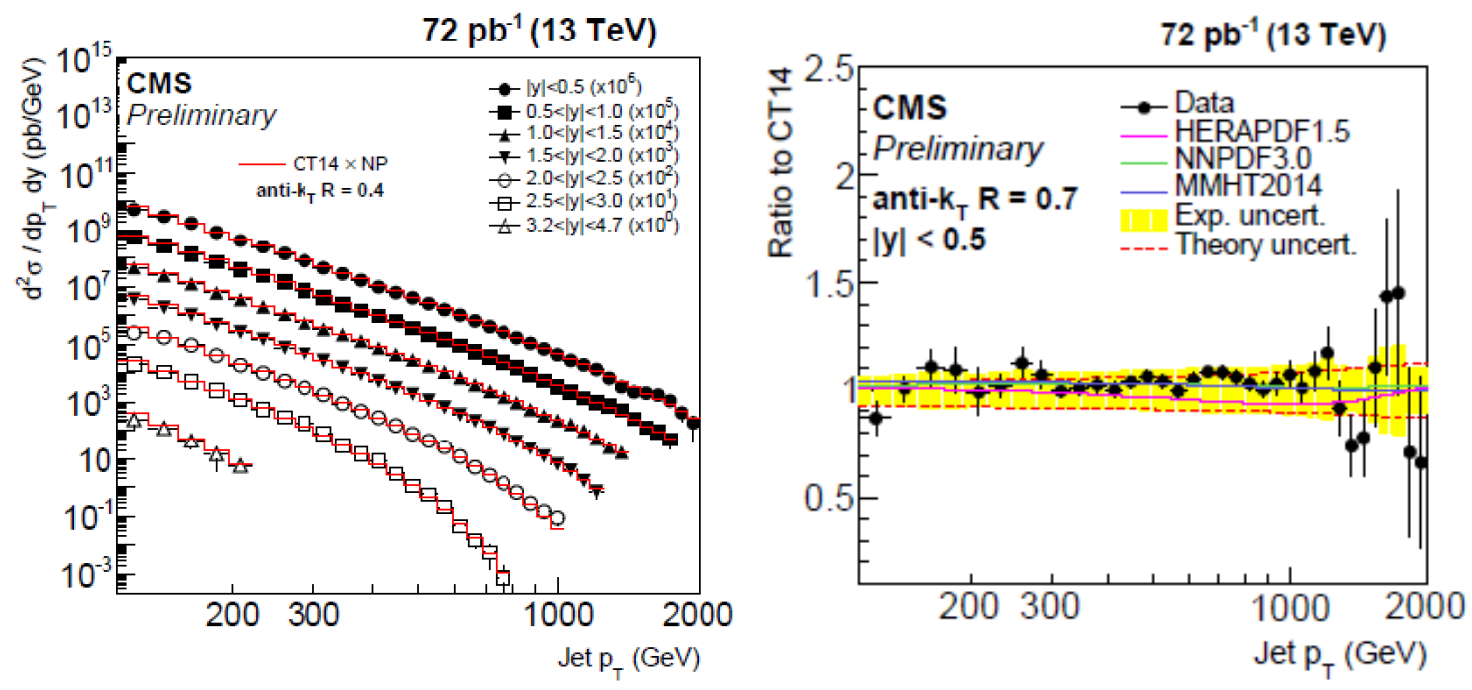

Figure 6: Inclusive jet cross sections as a function of the jet transverse momenta, for different intervals of pseudorapity (left); ratio of data to the predictions obtained from the CT14 set of pdfs, compared to predictions of alternative pdf's (right) [14].

\subsection{Jet Multiplicities}

Measurements of jet multiplicities constitute intrinsic tests of $\mathrm{QCD}$, as the probability for each additional jet is proportional to the value of the strong coupling, $\alpha_{s}$. Even more importantly, multijet events are a dominant background for many studies of and searches for heavy objects, like top quarks or new heavy bosons, respectively. These backgrounds must be known and determined, with highest confidence, from data themselves.

An important background to many BSM searches and Higgs boson studies is the associate production of a $\mathrm{Z}$ boson together with $n$ hadronic jets, where $n=0,1,2, \ldots$. Measurements of cross sections of $\mathrm{Z}+$ jets final states at $\sqrt{s}=13 \mathrm{TeV}$ are displayed in Fig. 7, for anti- $\mathrm{k}_{t}$ jets at $\mathrm{R}=$ 0.4 and $n=0$ to $4[16,17]$. The measurements closely resemble predictions made by QCD model calculations (comprising next-to-leading order perturbative QCD (NLO) plus parton shower Monte Carlo generators).

\section{Determinations of $\alpha_{s}$}

The strong coupling constant $\alpha_{s}$ is one of the most important, fundamental parameters of the SM of electroweak and strong interactions. Not being a physical observable itself, it is a quantity 

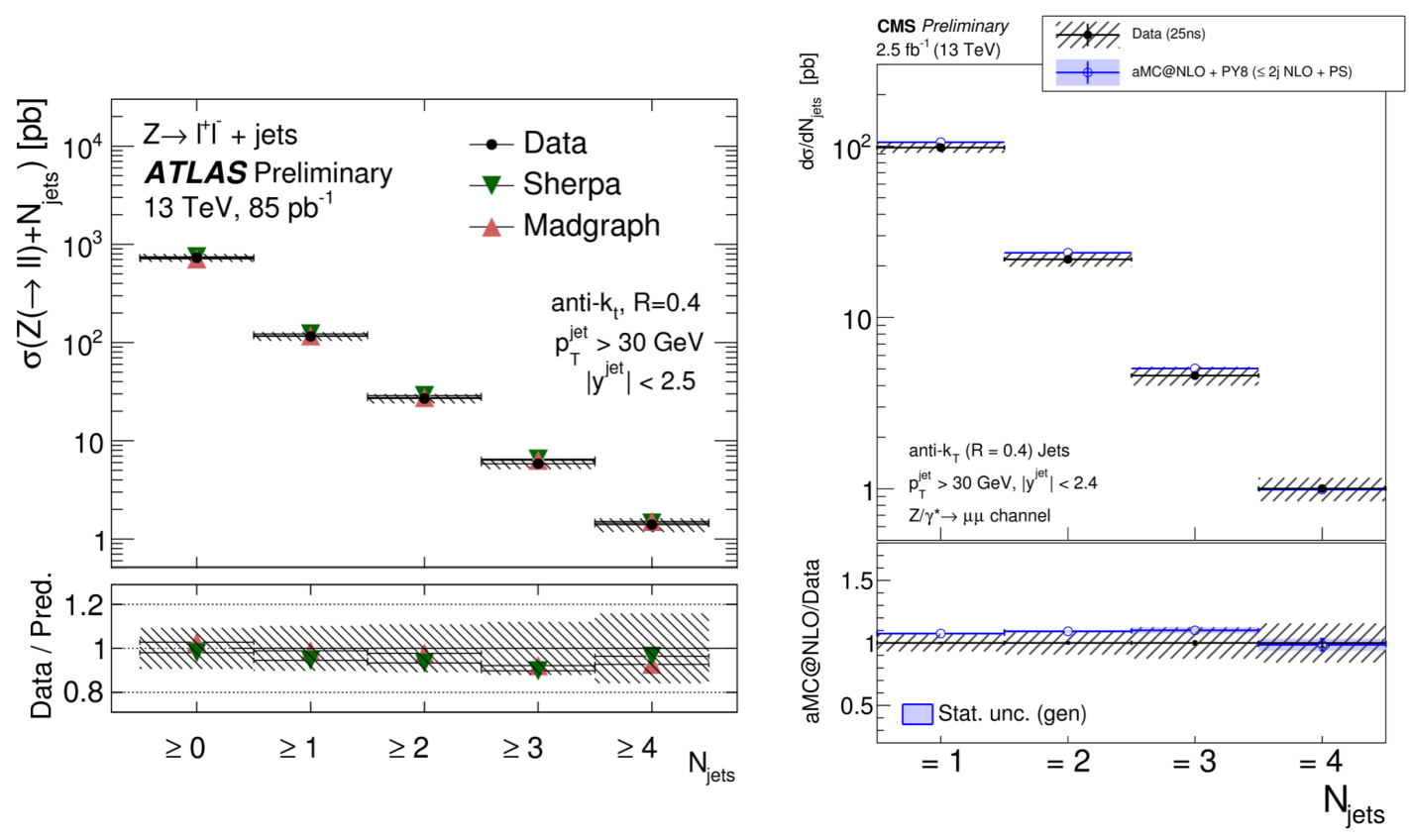

Figure 7: Associate production of jets and jet multiplicities in $\left(\mathrm{Z} \rightarrow \ell^{+} \ell^{-}\right)+$jet events.

defined in the context of Quantum Chromodynamics (QCD) perturbation theory [9], which enters predictions for experimentally measurable observables. Measurements of such observables, in turn, are used to determine the numerical value of $\alpha_{s}$. More spefically, since $\alpha_{s}$ is predicted - according to the concept of Asymptotic Freedom - to "run" with energy, values of $\alpha_{s}(Q)$ are determined, where $Q$ is relevant energy scale of data used in the determination ${ }^{3}$. The functional form of "running" is precisely predicted by the QCD $\beta$-function, such that each determination of $\alpha_{s}(Q)$ can be converted to a value of $\alpha_{s}\left(M_{Z}\right)$, at a common value of $Q=M_{Z}$, namely the mass of the Z-boson.

Determinations of $\alpha_{s}$ from hadron collider data, i.e. the Tevatron and the LHC, from the lepton-hadron collider HERA and the $e^{+} e^{-}$colliders LEP and PETRA are summarised in Fig. 8 [18]. All results from hadron colliders, except the one from $t \bar{t}$ production, and from HERA are derived in NLO QCD; results from $t \bar{t}$ production and LEP and PETRA are based on next-to-NLO (NNLO) QCD. Corresponding hadron collider results from ATLAS are also available [19] but are not reproduced in this figure; for a comprehensive summary of hadron collider results, see e.g. [9].

The LHC and Tevatron results can be combined and summarised as

$$
\alpha_{s}\left(M_{Z}\right)=0.1172 \pm 0.0059
$$

using combination methods as applied for the determination of the current world-average of $\alpha_{s}\left(M_{Z}\right)=$ $0.1181 \pm 0.0011$ [21]. The agreement with this world average, within errors, is excellent. Note, however, that the world average is determined from $\alpha_{s}$ determinations which are complete to at

\footnotetext{
${ }^{3}$ The choice of "relevant energy scale $Q$ " is somewhat arbitrary. In many case, $Q=\sqrt{s}$ is taken, but other choices like the masses or typical transverse momenta, $\mathrm{p}_{t}$, of analysed objects are also used, especially for processes at hadron or hadron-lepton colliders.
} 


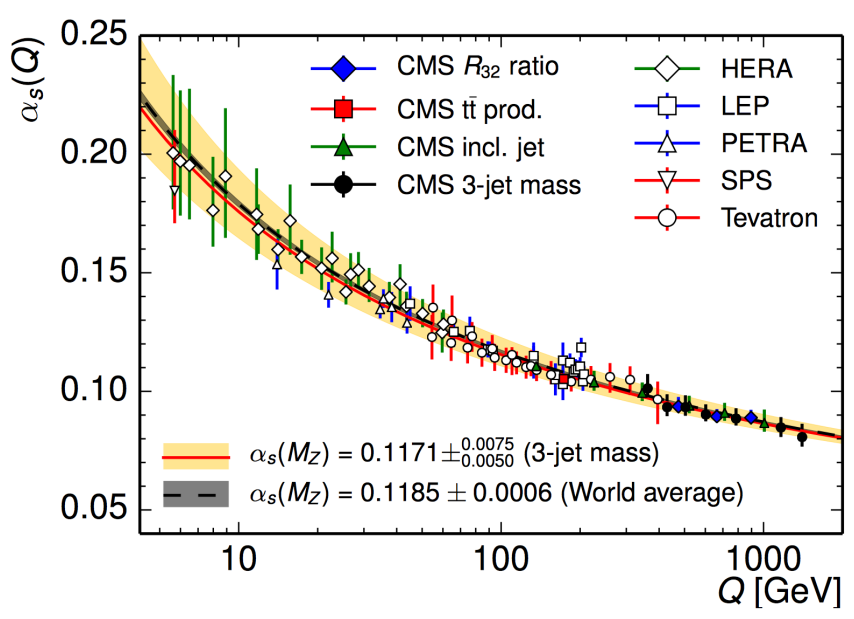

Figure 8: Measurements of $\alpha_{s}$ of CMS at the LHC and other collider experiments at lower $\mathrm{cm}$. energies [18]. Corresponding results from ATLAS are not included in this figure.

least NNLO perturbative QCD. Most of the results from hadron colliders are, however, in NLO only, with the exception of the result from $t \bar{t}$ production cross section [20], which in fact is included in the determination of the world average value.

It should also be noted that this particular $\alpha_{s}$ determination from $t \bar{t}$ production cross section, resulting in $\alpha_{s}\left(M_{Z}\right)=0.1151_{-0.0027}^{+0.0028}$, seems sytematically low, as more recent measurements of the $t \bar{t}$ production cross section, from ATLAS and CMS at 7, 8 and $13 \mathrm{TeV}$ c.m. energies, indicate larger values of $\alpha_{s}\left(M_{Z}\right)$ of about 0.1200 , see [22].

\section{Top Quark Physics}

The top quark, with a world average mass of $m_{t}=(173.3 \pm 0.8) \mathrm{GeV}$ [23] is the heaviest known fundamental particle. At hadron colliders, top quarks are predominantly produced in pairs $(t \bar{t})$, through the strong interaction process of gluon fusion, see the upper left graph in Fig. 9. Due to its large mass, it instantly decays and its properties are directly transferred to its decay products, namely a $\mathrm{W}$ boson and a b-quark, which in turn decay into well accessible and detectable final states comprising quarks and anti-quarks $q$ and $q^{\prime}$, charged leptons $\ell$ and neutrinos $v$. Single top quarks can be produced through electro-weak processes like those depicted in the lower panel of Fig. 9. Experimentally, top-quark pairs are detected and characterised by the decays of the two W bosons, which may decay hadronically, into two quarks, or leptonically, into a charged lepton plus its associated neutrino.

The upper right part of Fig. 9 gives the relative fractions branching fractions of top-quark decays into the classes of "alljets", "lepton+jets" and "dileptons". The class of "dijets" is the most abundant and focusses the entire top quark energy into detectable particles, however has the disadvantage of huge backgrounds from other hadronic processes at the collider. Final states with leptonic $\mathrm{W}$ decays offer clean experimental signatures, through energetic leptons, however suffer from the undetectable neutrino energy, especially in the case of "dileptons" where the missing energy is shared by two neutrinos. 


\section{Top Pair Branching Fractions}
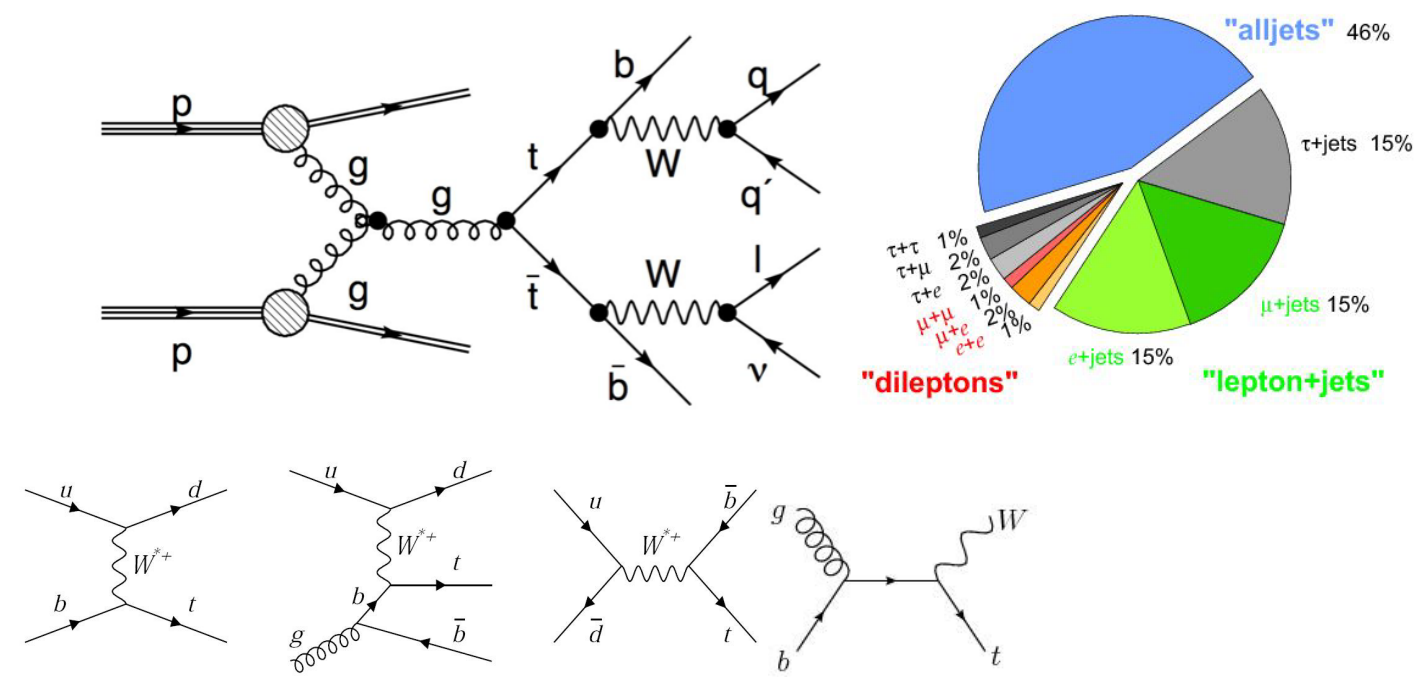

Figure 9: top left: top-quark pair production process through gluon fusion; top right: relative branching fractions of top-quark pair events; bottom: single top-quark production processes.

\subsection{Top quark production cross sections}

Measurements of top-pair and single-top production cross sections were obtained by ATLAS and CMS, at c.m. energies of 7, 8 and $13 \mathrm{TeV}$ [24, 25, 26, 27], see Fig. 10. The data are well described by the energy-dependent cross section predicted by calculations comprising QCD perturbation theory up to complete NNLO plus (next-)next-to-leading log approximations ((N)NLL).
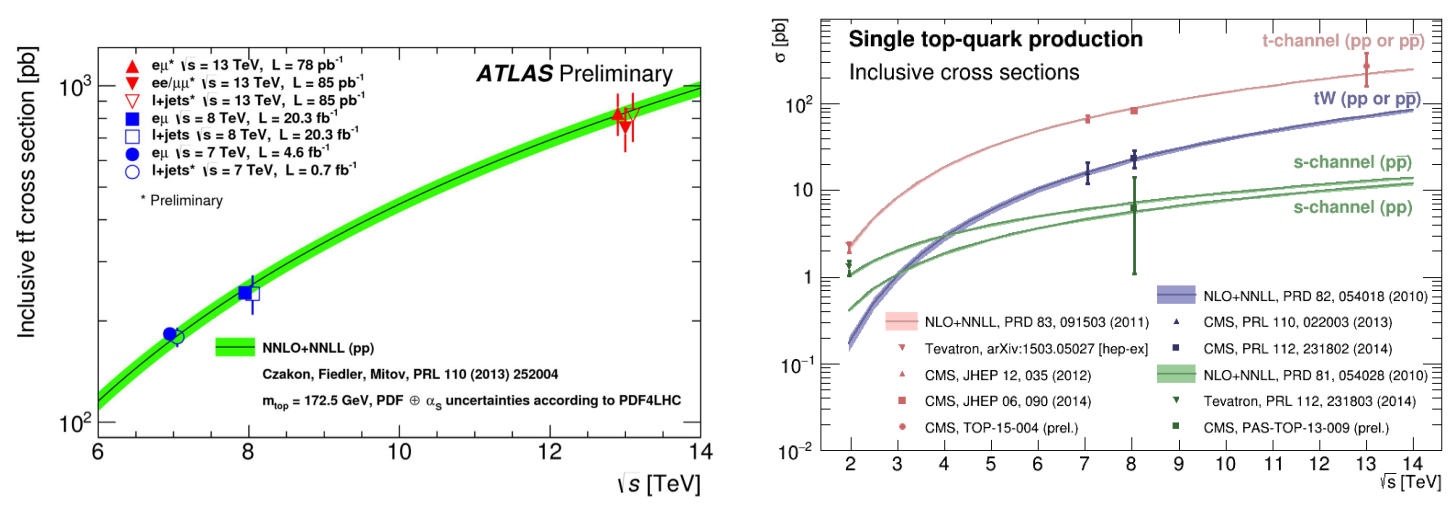

Figure 10: left: top-quark pair production cross sections at LHC energies [24]; right: single top-quark t-channel production cross sections in the Tevatron and LHC energy range [27].

\subsection{Top quark mass}

Precision measurements of the mass of the top-quark are performed using all principal classes of reconstructed $t \bar{t}$ events (dileptons, lepton\& jets, alljets), see Fig. 9. Most of these, commonly 


\begin{tabular}{|r|c|}
\hline & $m_{t}(\mathrm{GeV})$ \\
\hline world average (2014) [23] & $173.3 \pm 0.8$ \\
ATLAS (2015) [29] & $173.0 \pm 0.9$ \\
CMS (2015) [30] & $172.4 \pm 0.5$ \\
\hline
\end{tabular}

Table 1: Combinations of direct top-quark mass measurements.

called "direct" measurements, are based on template methods, i.e. the comparison of data distributions (reconstructed masses or related observables and estimators) to Monte-Carlo generated template distributions generated for a range of discrete top quark masses. The definition of bare masses of strongly-interacting objects like quarks require well defined theoretical frameworks and schemes, and any quantitative statement about the value of a quark mass must refer to the framework that is used to define it, see e.g. [28]. Monte-Carlo defined quark masses are not identical to those defined by theoretical frameworks, however the top quark mass utilized in Monte-Carlo generators is argued to be close to the so-called pole-mass, within an accuracy of $\mathscr{O}(1 \mathrm{GeV})$.

Summaries of direct top quark mass determinations by ATLAS [29] and CMS [30], at $\sqrt{s}=7$ and $8 \mathrm{TeV}$, are given in Fig. 11 and in Table 1. They are compared to the 2014 world average value of $m_{t}$ [23] and also to respective determinations at the Tevatron collider.
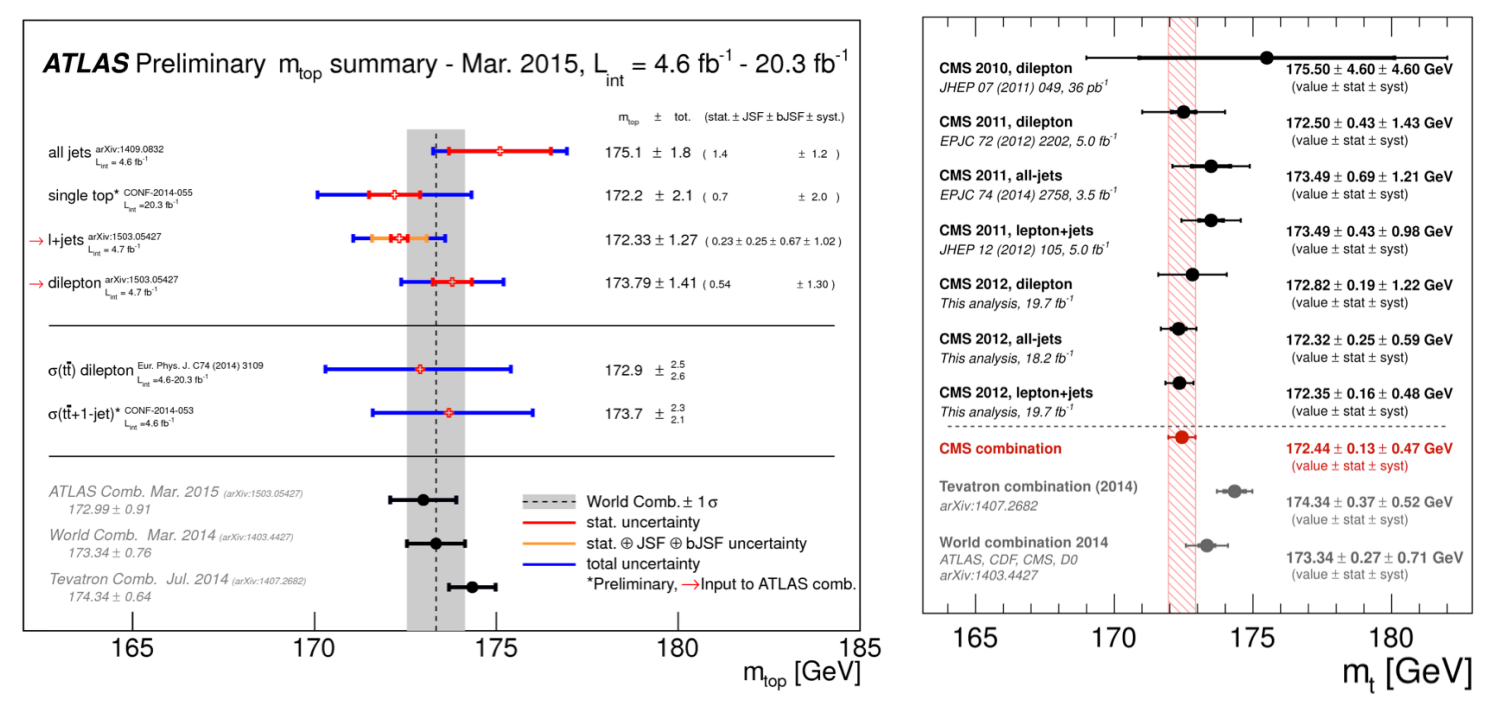

Figure 11: Summaries of direct measurements of the top-quark mass [29, 30].

The 2014 world average as well as the current average results of ATLAS and CMS agree well which each other, exhibiting overall experimental uncertainties of half a percent or less. This accuracy already requires to carefully relate Monte-Carlo defined top quark masses to those defined in theoretical frameworks. "Indirect" fits of the top quark pole mass from $t \bar{t}$ production cross sections become increasingly precise and agree, within their current uncertainties of $\approx \mathscr{O}(2 \mathrm{GeV})$, with the direct determinations of $m_{t}$, see Fig. 12 [31]. 


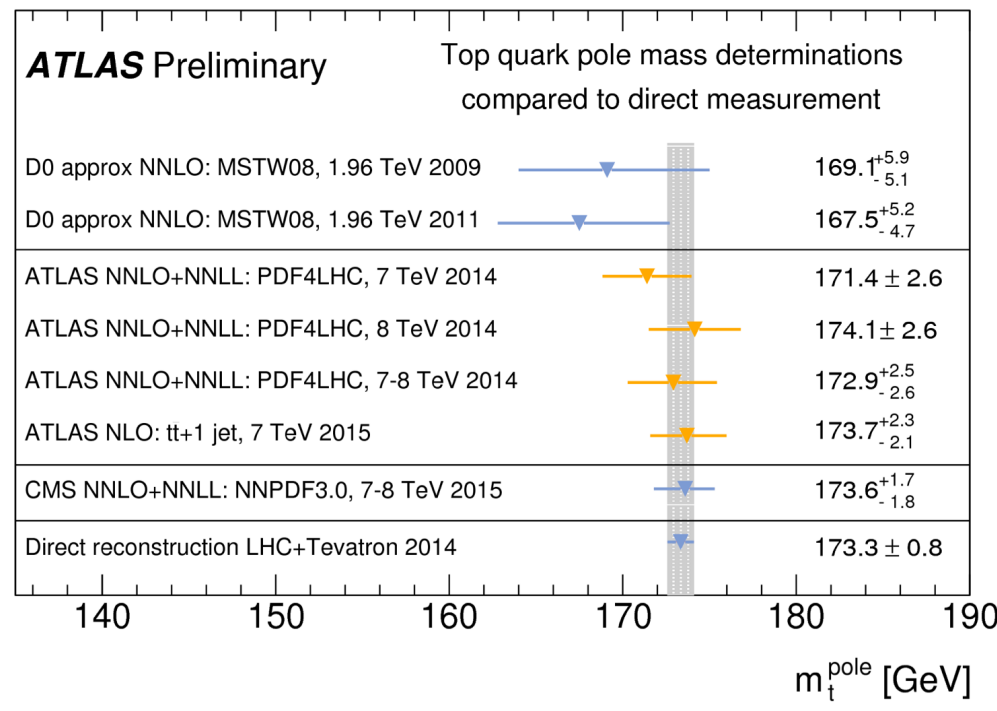

Figure 12: Summary of top-quark pole mass determinations [31].

\section{Heavy Ion Physics}

Traditionally, at the end of a year of high energy proton-proton collisions, the LHC is operated with beams of heavy ions $(\mathrm{Pb})$, in order to generate and study the hot and dense medium produced in high energy collisions of extended, bound systems of many nuclei. In 2015, LHC provided about $0.7 \mathrm{nb}^{-1}$ of Pb-Pb-collisions at $\sqrt{s_{N N}}=5.0 \mathrm{TeV}$, which corresponds to $\sqrt{s_{P b P b}}=1.1 \mathrm{PeV}$. At the time of this conference, however, no analyses based on these new data were released, such that here we refer to a selection of results obtained from previous heavy ion runs of the LHC, demonstrating some of the key features of the hot and dense medium, commonly phrased the quark-gluon plasma.

One of the observables studied in order to test and demonstrate the existence of collective phenomena in high energy heavy ion collisions is the jet nucleon modification factor $R_{A A}$, which is the ratio of (particle or energy) yields observed in heavy ion collisions, normalised by the same quantity observed in proton-proton collisions, at the same value of $\sqrt{s_{N N}}$. In Fig. 13, the modification factor $R_{A A}$ determined from $\mathrm{Pb}-\mathrm{Pb}$ and from p-p collsisions at $\sqrt{s_{N N}}=2.76 \mathrm{TeV}$, for hadron jets defined by the anti- $k_{\perp}$ jet algorithm with transverse momenta $p_{T}$ between 80 and $100 \mathrm{GeV}$, is shown as function of the pseudo-rapidity $y$ (top) and of the mean number of charged particles, $N_{\text {part }}$ (bottom) [32]. Together these distributions show that there is a suppression of hadron jets in central heavy ion collisions, which is largely independent of the rapidity (i.e. the polar angle) of jets but which significantly depends on the "centrality" of the collision, i.e. the degree of overlap of the two colliding objects. As expected for a colour charge shielding effect of a dense quark-gluon-plasma, the suppression of jets for the $10 \%$ most central collisions is $50 \%$ or more $\left(R_{A A}<50 \%\right)$, while for the least central collisions, at low $N_{\text {part }}$, the suppression effect is weaker $\left(R_{A A} \approx 80 \%\right)$.

Another observation demonstrates the different behavior of phenomena as function of the centrality of heavy-ion collisions, in comparison with reference p-p data: as demonstrated in Fig. 14, 

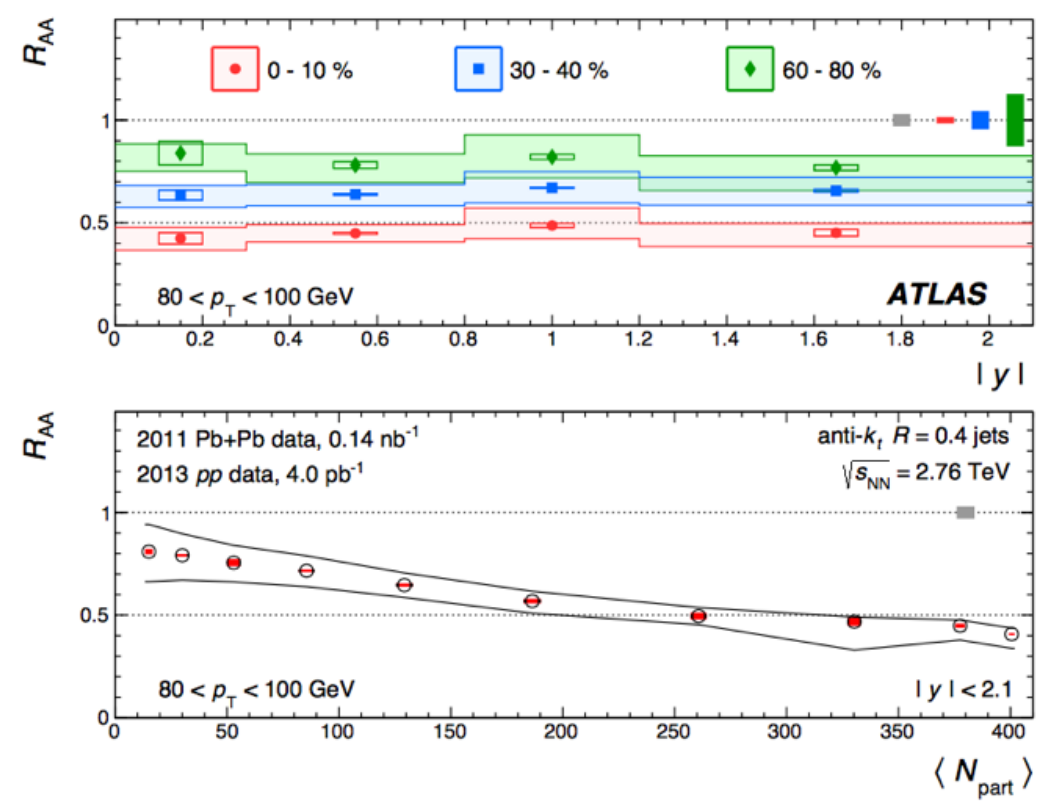

Figure 13: The jet modification factor $R_{A A}$ [32]. Top: as a function of pseudo-rapidity $y$, for 0-10, 30-40 and $60-80 \%$ of the events with highest centrality of the collision. Bottom: as a function of the number of charged particles produced in the collision (large $N_{\text {part }}$ correspond to high centrality, small $N_{\text {part }}$ indicate peripheral collisions).

there is a significant enhancement of the fragmentation function of charged particles with $\mathrm{p}_{T}<$ $3 \mathrm{GeV}$ in the most central $\mathrm{Pb}-\mathrm{Pb}$-collisions (right-most column), i.e. charged particles are significantly decelerated in the hot and dense medium [33].

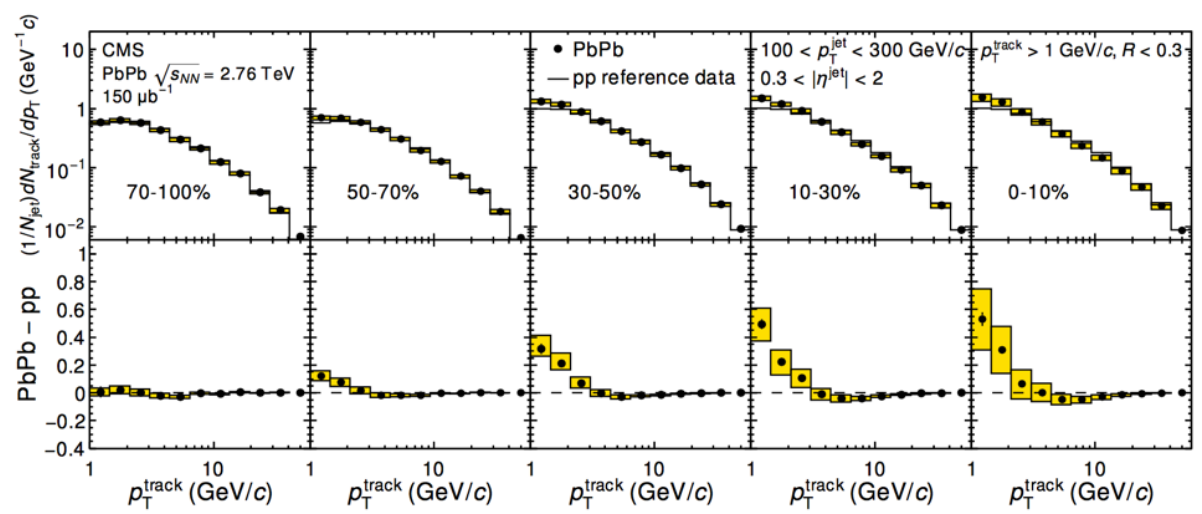

Figure 14: Transverse momentum distribution of charged particles inside jet cones $\left(100<p_{T}^{\text {jet }}<300 \mathrm{GeV}\right)$, for events with low (left column) up to the highest (right column) degrees of centrality [33].

\section{Searches for New Phenomena}

The Standard Model of particles, although proven to successfully describe the wealth of data 
from high energy particle collisions, leaves open many fundamental questions, like the origin and nature of dark matter and dark energy, the asymmetry between matter and antimatter in the universe, the origin of neutrino masses, the difference between energy scales of electroweak symmetry breaking, grand unification and the Planck mass, and many other issues which range from subtle to universal. There exist many ideas to extend or supplement the Standard Model, like Supersymmetry (SUSY) and extra space dimensions, see e.g. the corresponding review articles in [10]. No experimental verification of the relevance of any of this models in nature, however, has so far been demonstrated. It is therefor one of the utmost tasks of the LHC and its experiments to generate and study collision data at the energy frontier in terms of signatures for New Physics beyond the Standard Model (BSM). Experimental searches for BSM physics concentrate on both, the direct observation of new and yet unknown particles, presumably at high and so far unexplored mass scales, and on other, mostly kinematic effects, which are governed by virtual corrections through new particles at even higher mass scales which are not yet accessible today.

\subsection{Global Summary of Run-I Results}

Both ATLAS and CMS have performed large numbers of explicit searches for new particles and phenomena, however so far no significant signal of any BSM physics has been observed. These searches, if negative, usually lead to exclusion limits for such new physics effects, at a given level of confidence. Both ATLAS and CMS have performed a large number of dedicated searches, leading - in the absence of significant positive signals - to many exclusion limits which, however, are model dependent and must be interpreted with appropriate care.

A summary of the most important exclusion limits from ATLAS using run-1 data at $\sqrt{s}=7$ and $8 \mathrm{TeV}$, for a large variety of BSM models and predictions, is given in Fig. 15 [34]. Similar results are available from CMS [35]. Corresponding summaries of exclusion limits for Supersymmetry (SUSY) are given in a separate presentation at this symposium [3].

The currently achieved exclusion limits, in almost all cases, reach masses of $\mathscr{O}(1 \mathrm{TeV})$ and above, providing significant constraints on a large variety of BSM models and expectations. At the time of this symposium, some of these searches have been extended to the most recent run-2 data at $\sqrt{s}=13 \mathrm{TeV}$; details of some of the key analyses, some of which provide results already exceeding those from run-1, are given in the following subsections.

\subsection{Dijet Resonant Searches}

Many models of BSM physics predict new particles and their immediate decays into two high energetic quarks and/or gluons, giving rise to resonances in spectra of pair masses of hadronic jets. Examples for such speculative objects are new color octet scalars, excited quarks, scalar quarks or quantum black holes. Dijet mass spectra of both ATLAS [36] and CMS [37] are presented in Fig. 16, using their most recent data at $\sqrt{s}=13 \mathrm{TeV}$. The data exhibit a smooth and steeply falling behavior without any significant deviation that would be consistent with a dijet resonance expected for new heavy objects, providing exclusion limits e.g. for excited quarks with masses below $\approx 5 \mathrm{TeV}$, and for quantum black holes below $\approx 5$ to $8 \mathrm{TeV}$, depending on the detailed parameters of the models. 


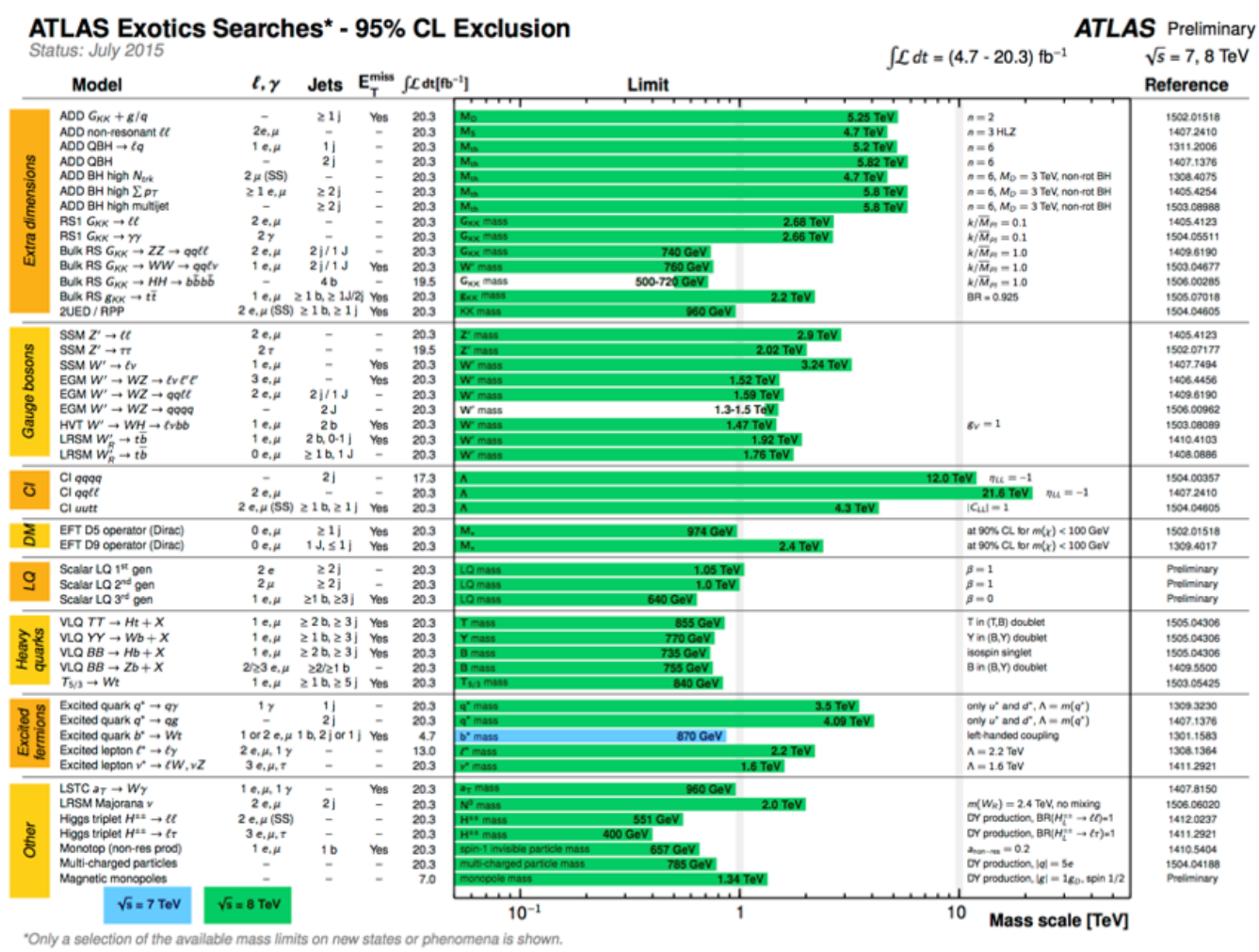

Figure 15: Reach of ATLAS searches for new phenomena other than Supersymmetry [34]. Only a representative selection of the available results is shown.

\subsection{Search for Heavy Gauge Bosons}

New heavy gauge bosons, commonly denoted as $W^{\prime}$ and $Z^{\prime}$, are another class of objects which are subject of many searches for BSM physics. Two of the most recent results from ATLAS and CMS, from a dilepton resonance search [38] and from a study of leptons plus missing energy [39], are displayed in Fig. 17. In both cases, the data follow the expected background from Standard Model processes, without any sign for significant contributions from effects expected for the presence and decays of heavy gauge bosons. The corresponding exclusion limits are up to $3.4 \mathrm{TeV}$ for $Z^{\prime}$, and $4.4 \mathrm{TeV}$ for $W^{\prime}$.

\subsection{Diboson Resonant Searches}

One modest excess of data in searches performed using the run-1 data of ATLAS was previously reported to be seen in a study of diboson ( $\mathrm{Z}$ and/or $\mathrm{W}$ ) resonances in the fully hadronic decay channel. Here, both highly boosted bosons decay into jets of hadrons giving rise to a substructure of two jets for each of the two bosons, see Fig.18 (left and middle) [40]. The excess observed around masses of $2 \mathrm{TeV}$ was reported to exhibit a 3.4 standard deviations $(\sigma)$ local and of $2.5 \sigma$ global significance. 

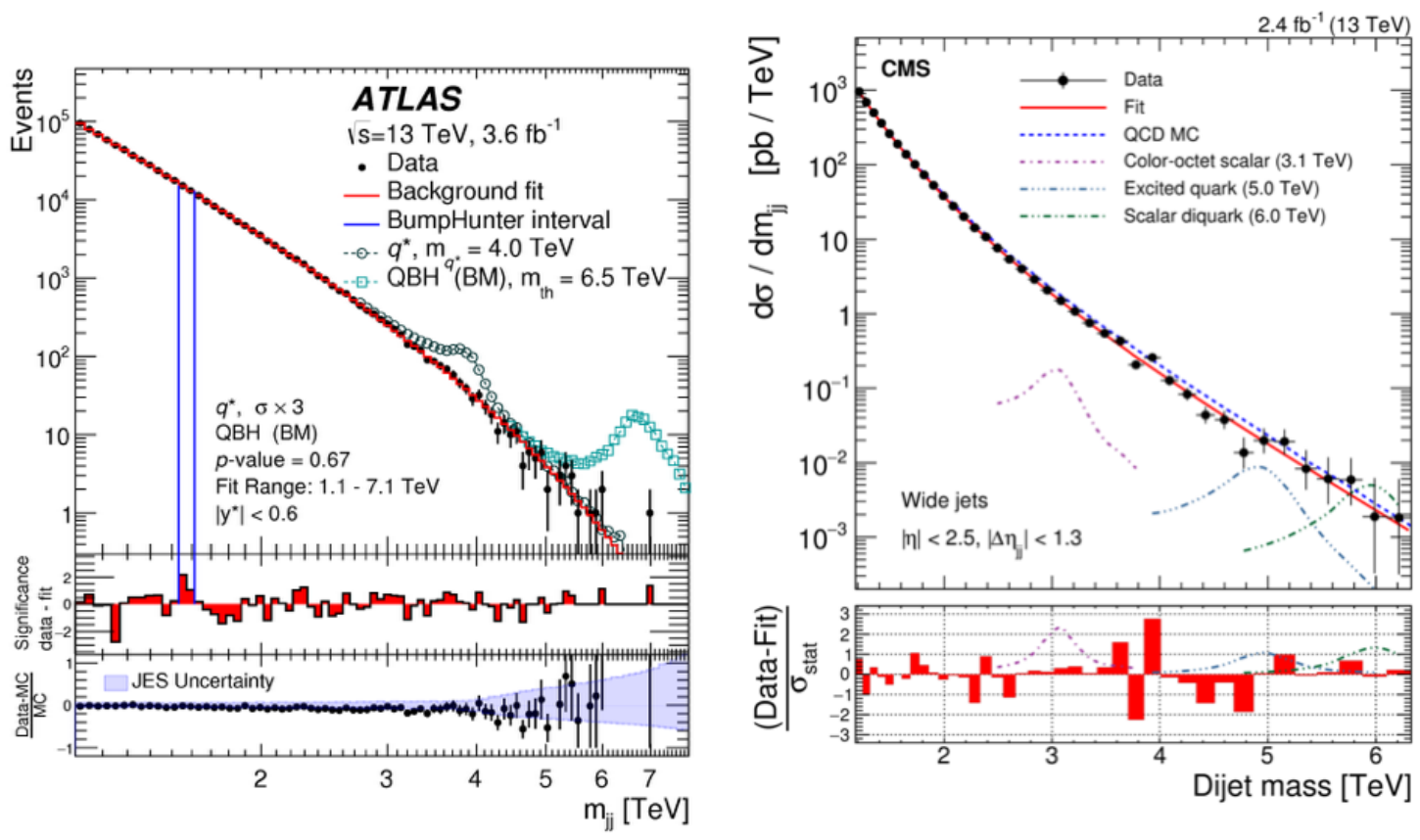

Figure 16: Dijet invariant mass distributions at $\sqrt{s}=13 \mathrm{TeV}[36,37]$.
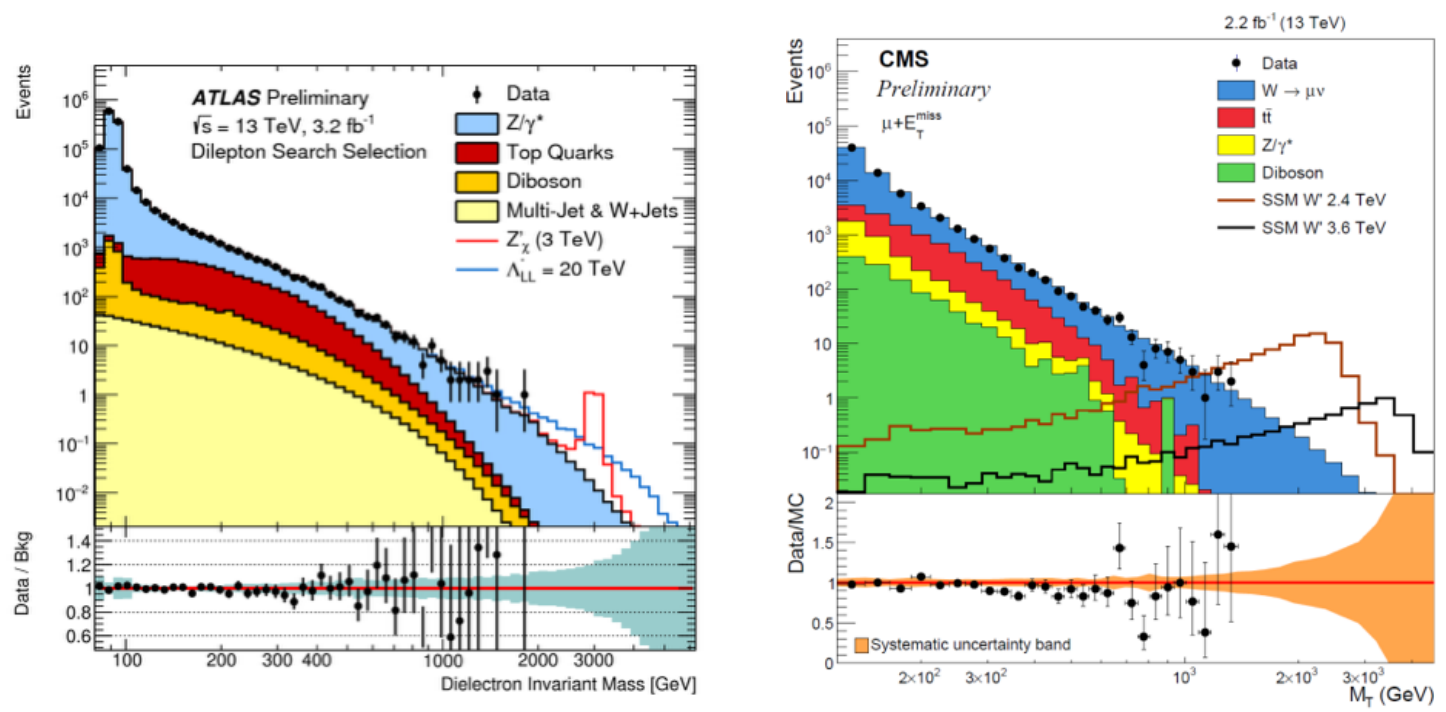

Figure 17: Dilepton invariant mass distribution [38] (left) and the transverse lepton-and-missing-energy invariant mass distribution [39] (right) in searches for new heavy bosons, Z and W, respectively. 
The result of the same analysis, applied to the new data of run-2, are displayed in the right part of Fig. 18, where no excess is being observed. The sensitivity of the new data, however, is still too low for a conclusive probe, such that a definitive answer on the existence of a new diboson resonance around $2 \mathrm{TeV}$ has to await further data at $\sqrt{s}=13 \mathrm{TeV}$ or higher.
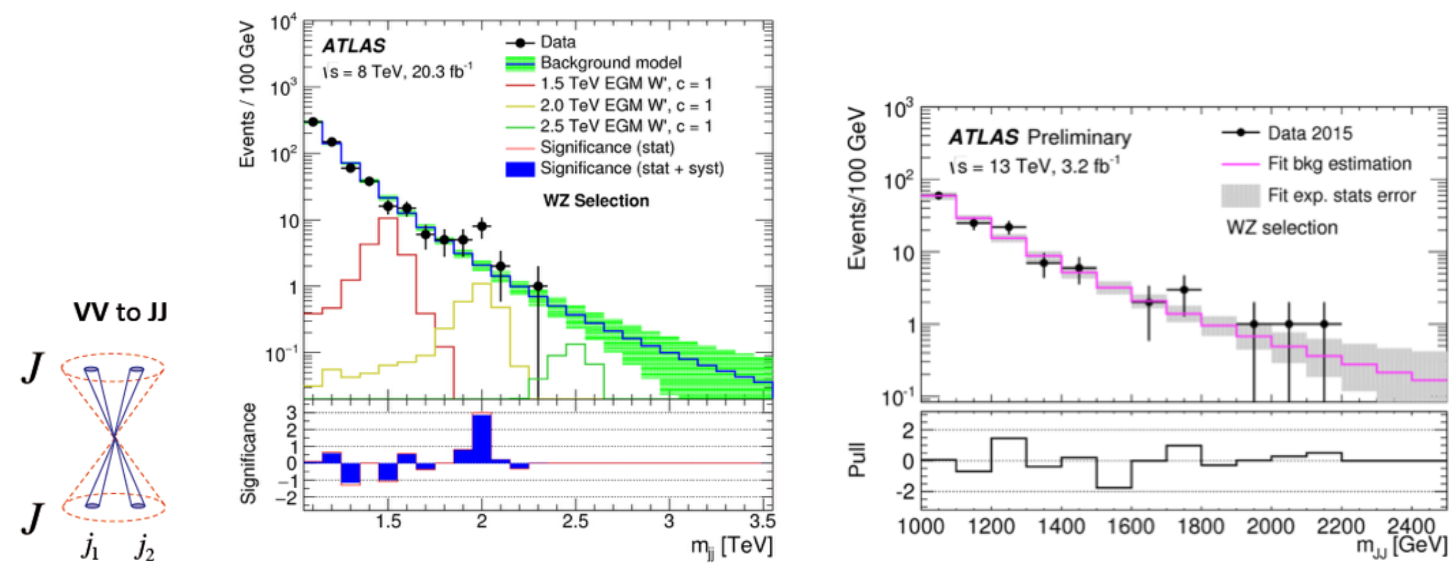

Figure 18: Two highly boosted bosons, reconstructed as (fat) jets $J$, with a substructure of 2 slim jets (j) (left most graphic). Diboson invariant mass distribution measured at $\sqrt{s}=8 \mathrm{TeV}$ [40] (middle); same at $\sqrt{s}$ $=13 \mathrm{TeV}$ (right).

\subsection{Diphoton Resonant Searches}

The largest excitement caused by early results from run-II data was an excess signal seen in the invariant two-photon mass spectrum around $750 \mathrm{GeV}$, reported by both ATLAS [41] and CMS [42], see Fig. 19. Details and results of the two analyses are summarised and compared in Table 2. The distributions of local p-values and significances, obtained when comparing the data with expected background (no signal) distributions, are displayed in Fig. 20.

While the distributions and some of the local p-values look very promising, the overall, and especially the global significances are still far from being at the $5 \sigma$ level required for claiming a discovery. No official combination of ATLAS and CMS results has been released so far.

This unexpected "bump" in the di-photon spectrum at high masses around $750 \mathrm{GeV}$ is not predicted by the Standard Model of particle physics, and would be - if confirmed by the data expected from the ongoing run-2 in 2016 and beyond - the first indication for a new massive particle beyond the Standard Model. The nature of a new object with properties as suggested by these initial studies is not clear at all, as is demonstrated by many hundreds of theory papers that have been published on this issue. Speculations about the origin and nature of such a new particle include a new Higgs-like boson, a particle from within a specially tuned version of Supersymmetry, a particle linked to a new kind of strong, fundamental force, and a particle as decay product from even more massive, new particles.

\section{Summary}

Run-2 of the Large Hadron Collider, at $\sqrt{s}=13 \mathrm{GeV}$, has successfully started in 2015. The 

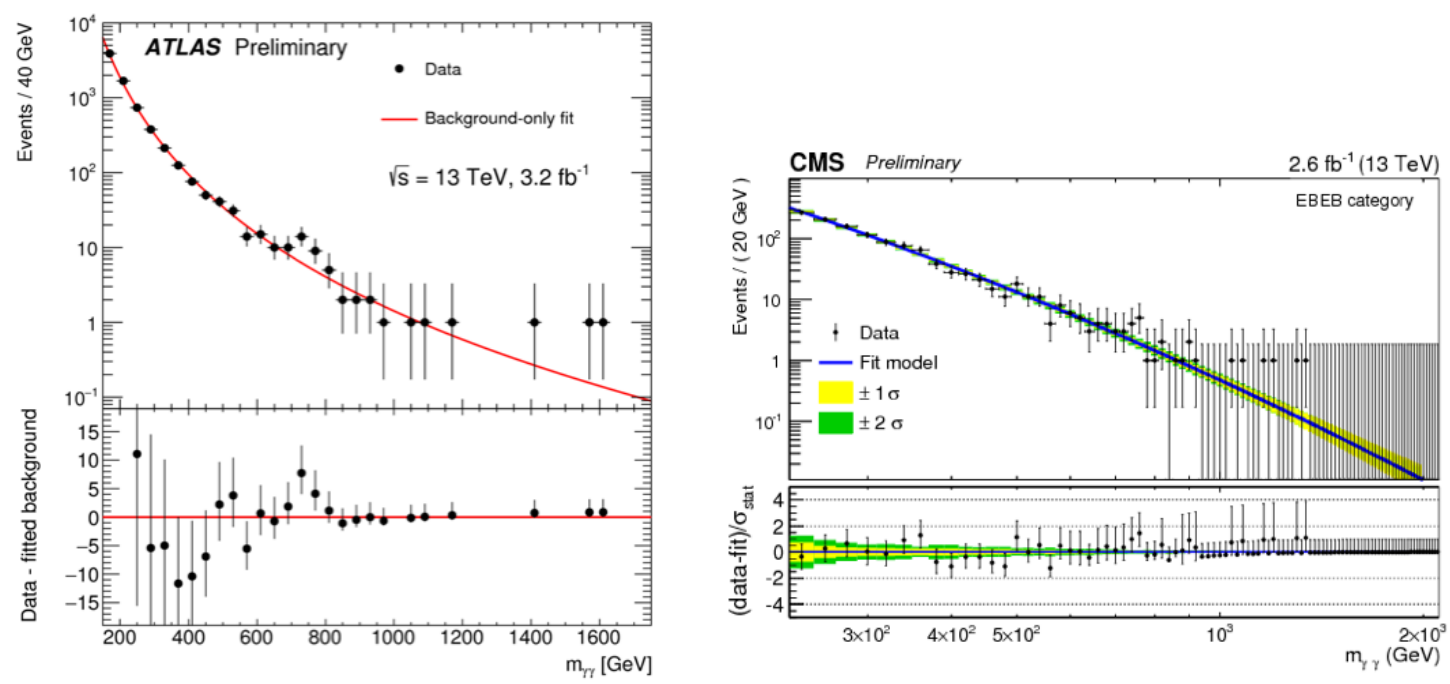

Figure 19: Invariant di-photon mass distributions obtained at $\sqrt{s}=13 \mathrm{TeV}$ by ATLAS [41] (left) and CMS (right). For CMS, the distribution for both photons in the barrel part of the detector is shown; a separate distribution for events with one photon in the endcap is also available [42].

\begin{tabular}{|r||l|l|}
\hline \multicolumn{1}{|c||}{ quantity } & ATLAS [41] & CMS [42] \\
\hline \hline initial search for & spin-0 object from gg-fusion & Randall-Sundrum gravitons \\
\hline data sample & $3.2 \mathrm{fb}^{-1}$ & $2.6 \mathrm{fb}^{-1}$ \\
\hline exp. mass resolution & $\approx 1 \%$ & $1.1 \% / 1.8 \%$ (barrel/endcap) \\
\hline bin size (mass plot) & $40 \mathrm{GeV}$ & $20 \mathrm{GeV}$ \\
\hline local p-value significance & $3.6 \sigma$ at $750 \mathrm{GeV}$ & $2.6 \sigma$ at $760 \mathrm{GeV}$ \\
\hline global p-value significance & $2.0 \sigma$ & $<1.2 \sigma$ \\
for fit range & $200-2000 \mathrm{GeV}$ & $500-5400 \mathrm{GeV}$ \\
and assumed width & narrow $(0.004 \mathrm{GeV})$ & narrow $(0.1 \mathrm{GeV})$ \\
\hline local / global significance & $3.9 / 2.3 \sigma$ & $2.0 /<1.0 \sigma$ \\
at large width & $\Gamma=45 \mathrm{GeV}$ (best fit) & $\Gamma=42 \mathrm{GeV}$ \\
\hline 8 TeV data consistency check & consistent at $2.2 \sigma$ level & combined signif.: 3.1 / <1.7 $\sigma$ \\
\hline
\end{tabular}

Table 2: Summary and comparison of details of the di-photon resonance search of ATLAS and CMS (status: January 2016).

acquired integrated luminosity of up to $4 \mathrm{fb}^{-1}$, by each of the large multi-purpose detectors ATLAS and CMS, provides novel insights into the physics at highest collider energies, and is expected to grow up to $100 \mathrm{fb}^{-1}$ by 2018. The results from 2015 data significantly extend and - in some cases - even exceed those obtained from the data of run-1 (at $\sqrt{s}=7$ and $8 \mathrm{GeV}$ ). In this presentation, global properties and precision results of fundamental parameters of the Standard Model, like the mass and production cross section of the top quark and of the strong coupling $\alpha_{s}$ are summarised. With these parameters, Quantum Chromodynamics (QCD) - the gauge theory of the Strong Interaction within the Standard Model of particle physics - provides an excellent description and reliable 

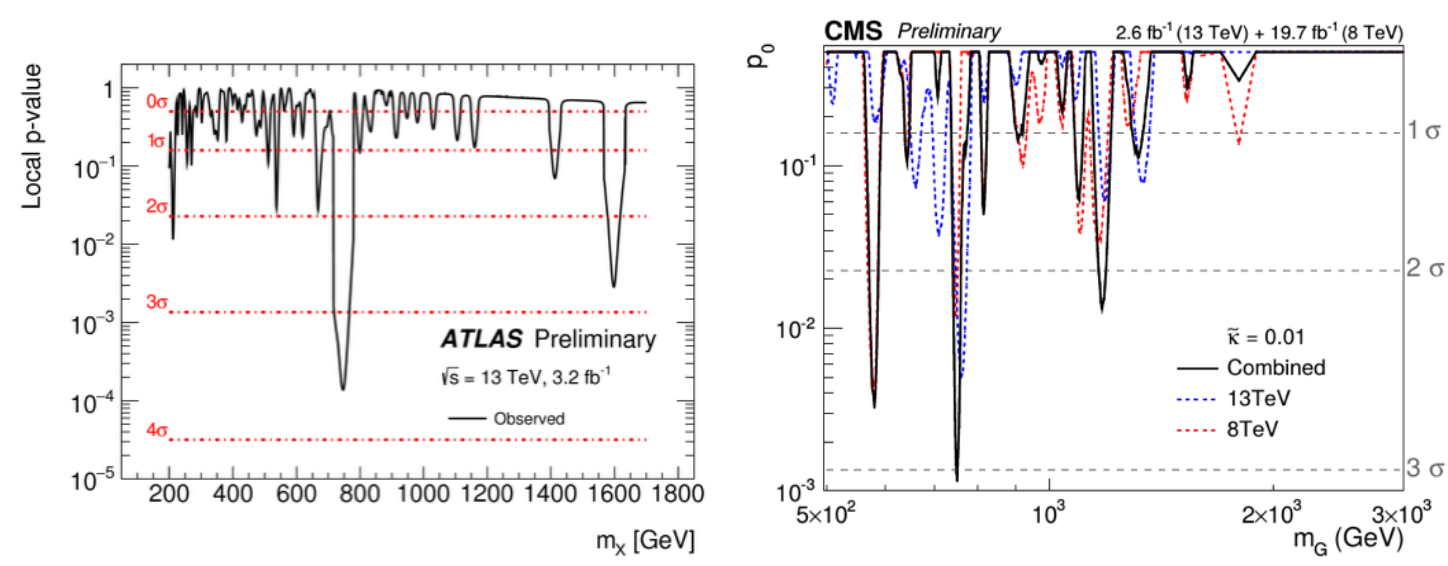

Figure 20: Local p-values and significances of deviations of the 2-photon invariant mass distribution from SM expectations, for ATLAS data at $\sqrt{2}=13 \mathrm{TeV}$ data (left); same for CMS, also for data at $\sqrt{s}=8 \mathrm{TeV}$ and a combination of 8 and $13 \mathrm{TeV}$ results.

predictions of high energy phenomena at LHC. So far, no significant deviations from the expectations of the Standard Model were seen. In explicit searches for signals of new physics beyond the Standard Model, modest excess signals seen in distributions of di-boson masses (run-1) were observed. More data statistics will be needed to decide between statistical fluctuations or genuine signals for new physics. The same conclusion holds for the "bump" in the di-photon invariant mass observed around $750 \mathrm{GeV}$ in the first year's data of run-2, which boosts hopes and imagination for the near future.

\section{Acknowledgments}

I wish to cordially thank the organisers of this meeting for invitation and for providing a very pleasant and inspiring environment at this meeting. The results presented above are the outcome of a huge and collaborative effort of many thousand scientists, engineers and technicians around the world who coherently designed, built and operated LHC and the large particle detectors ATLAS and CMS; without all their inspiration, genius and dedication, the results and this presentation would not have been possible.

\section{References}

[1] L. Evans, P. Bryant, LHC Machine, J. Inst. 3 (2008) 88-89.

[2] https://twiki.cern.ch/twiki/bin/view/AtlasPublic/LuminosityPublicResultsRun2.

[3] P. Azzurri, this conference.

[4] G. Aad et al. (ATLAS Collab.), The ATLAS Experiment at the CERN Large Hadron Collider, JINST 3 (2008) S08003.

[5] S. Chatrchyan et al. (CMS Collab.), The CMS experiment at the CERN LHC, JINST 3, (2008) S08004. 
[6] J. M. Campbell, J.W. Huston, W.J. Stirling, Hard Interactions of Quarks and Gluons: A Primer for LHC Physics, Rept.Prog.Phys. 70 (2007) 89; hep-ph/0611148

[7] Graphics taken from: Jim Olsen, LHC Jamboree at CERN, Dec 15, 2015.

[8] ATLAS Collab., Measurement of the Inelastic Proton-Proton Cross Section at $\sqrt{s}=13$ TeV with the ATLAS Detector at the LHC, ATLAS-Conf-2015-038.

[9] S: Bethke, G. Dissertori and G. Salam, Quantum Chromodynamics in: [10].

[10] K.A. Olive et al. (Particle Data Group), Chin.Phys. C38 (2014).

[11] V. Khachatryan et al. (CMS Collab.), Pseudorapidity distribution of charged hadrons in proton-proton collisions at $\sqrt{s}=13$ TeV, Phys.Lett. B 751 (2015) 143.

[12] V. Khachatryan et al. (CMS Collab)., Measurement of long-range near-side two-particle angular correlations in pp collisions at sqrts = 13 TeV, Phys.Rev.Lett. 116 (2016) no.17, 172302.

[13] G. Aad et al. (ATLAS Collab.), Observation of Long-Range Elliptic Azimuthal Anisotropies in $\sqrt{s}=$ 13 and $2.76 \mathrm{TeV}$ pp Collisions with the ATLAS Detector Phys.Rev.Lett. 116 (2016) no.17, 172301

[14] CMS Collab., Measurement of the double-differential inclusive jet cross section at sqrts $=13 \mathrm{TeV}$, CMS-PAS-SMP-15-007.

[15] M. Cacciari, G.P. Salam and G. Soyez, The Anti-k(t) jet clustering algorithm, JHEP 0804 (2008) 063.

[16] ATLAS Collab., Measurement of the Production Cross Sections of a Z Boson in Association with Jets in pp collisions at $\sqrt{s}=13 \mathrm{TeV}$ with the ATLAS Detector, ATLAS-CONF-2015-041.

[17] CMS Collab., Measurement of the differential cross section of Z boson production in association with jets in proton-proton collisions at $\sqrt{s}=13 \mathrm{TeV}, C M S-P A S-S M P-15-010$.

[18] V. Khachatryan et al. (CMS Collab.), Measurement of the inclusive 3-jet production differential cross section in proton-proton collisions at $7 \mathrm{TeV}$ and determination of the strong coupling constant in the TeV range, Eur. Phys. J. C75 (2015) 5, 186

[19] G. Aad et al. (ATLAS Collab.), Measurement of transverse energy-energy correlations in multi-jet events in pp collisions at $\sqrt{s}=7 \mathrm{TeV}$ using the ATLAS detector and determination of the strong coupling constant $\alpha_{S}\left(M_{Z}\right)$, Phys.Lett. B750 (2015) 427.

[20] S. Chatrchyan et al., (CMS Collab.), Determination of the top-quark pole mass and strong coupling constant from the t $t$-bar production cross section in pp collisions at $\sqrt{s}=7$ TeV, Phys.Lett. B728 (2014) 496.

[21] see the 2016 version of [9], to be published in Chin.Phys. C49 (2016).

[22] S. Bethke, G. Dissertori, T. Klijnsma and G. Salam, $\alpha_{s}$ from top-pair cross sections, in: workshop on High-precision $\alpha_{s}$ measurements from LHC to FCC-ee, arXiv:1512.05194.

[23] The ATLAS, CDF, CMS and D0 Collaborations, First combination of Tevatron and LHC measurements of the top-quark mass, arXiv:1403.4427.

[24] ATLAS Collab., Measurements of the t $\bar{t}$ production cross-section in the dilepton and lepton-plus-jets channels and of the ratio of the $t \bar{t}$ and $Z$ boson cross-sections in pp collisions at $\sqrt{s}=13$ TeV with the ATLAS detector, ATLAS-CONF-2015-049.

[25] V. Khachatryan et al. (CMS Collab.), Measurement of the top quark pair production cross section in proton-proton collisions at $\sqrt{s}=13 \mathrm{TeV}$, arXiv:1510.05302. 
[26] ATLAS Collab., Measurement of the inclusive cross-section of single top-quark t-channel production in pp collisions at $\sqrt{s}=13 \mathrm{TeV}$, ATLAS-CONF-2015-079.

[27] CMS Collab., Measurement of the t-channel single top-quark cross section at $13 \mathrm{TeV}$, CMS-PAS-TOP-15-004.

[28] A. Manohar and C. Sachrajda, Quark Masses in: [10].

[29] G. Aad et al. (ATLAS Collab.), Measurement of the top quark mass in the $t \bar{t} \rightarrow$ lepton+jets and $t \bar{t} \rightarrow$ dilepton channels using $\sqrt{s}=7$ TeV ATLAS data, Eur. Phys. J. C (2015) 75:330 and updates submitted to Moriond 2015 conferences.

[30] V. Khachatryan et al. (CMS Collab.), Measurement of the top quark mass using proton-proton data at $\sqrt{s}=7$ and 8 TeV, Phys.Rev. D93 (2016) no.7, 072004.

[31] G. Aad et al. (ATLAS Collab.), Determination of the top-quark pole mass using ttbar+1-jet events collected with the ATLAS experiment in 7 TeV pp collisions, JHEP 10 (2015) 121 and updates prepared for the 2015 Moriond conferences.

[32] G. Aad et al. (ATLAS Collab.), Measurements of the nuclear modification factor for jets in $\mathrm{Pb}+\mathrm{Pb}$ collisions at $\sqrt{s_{N N}}=2.76$ TeV with the ATLAS detector, PRL 114 (2015) 072302.

[33] S. Chatrchyan et al. (CMS Collab.), Measurement of jet fragmentation in $\mathrm{PbPb}$ and pp collisions at $\sqrt{s_{N N}}=2.76$ TeV, Phys. Rev. C 90 (2014) 024908.

[34] ATLAS Collab., Summary of Exotics WG, status of July 2013.

[35] CMS Collab., Exotica Physics Group Summary, status of March 2015.

[36] G. Aad et al. (ATLAS Collab.), Search for New Phenomena in Dijet Mass and Angular Distributions from pp Collisions at $\sqrt{s}=13 \mathrm{TeV}$ with the ATLAS Detector, Phys. Lett. B754 (2016) 302.

[37] V. Khachatryan et al. (CMS Collab.), Search for narrow resonances decaying to dijets in proton-proton collisions at $\sqrt{s}=13 \mathrm{TeV}$, Phys. Rev. Lett. 116, 071801 (2016).

[38] G. Aad et al. (ATLAS Collab.), Search for high-mass new phenomena in the dilepton final state using proton-proton collisions at $\sqrt{s}=13 \mathrm{TeV}$ with the ATLAS detector, arXiv:1607.03669

[39] CMS Collab., Search for SSM W' production, in the lepton+MET final state at a center-of-mass energy of $13 \mathrm{TeV}$, CMS-PAS-EXO-15-006.

[40] ATLAS Collab., Search for resonances with boson-tagged jets in $3.2 \mathrm{fb}^{-1}$ of p p collisions at $\sqrt{\mathrm{s}}=13$ TeV collected with the ATLAS detector, ATLAS-CONF-2015-073.

[41] ATLAS Collab., Search for resonances decaying to photon pairs in $3.2 \mathrm{fb}^{-1}$ of pp collisions at $\sqrt{\mathrm{s}}=$ $13 \mathrm{TeV}$ with the ATLAS detector ATLAS-CONF-2015-081.

[42] CMS Collab., Search for new physics in high mass diphoton events in proton-proton collisions at $\sqrt{s}$ $=13 \mathrm{TeV}, \mathrm{CMS}$ PAS EXO-15-004. 